\title{
Fluorodeoxyglucose positron emission tomography findings in NMDA receptor antibody encephalitis
}

\author{
Pedro Enrique Jiménez Caballero
}

A 28-year-old woman presented with a seven day history of insomnia, and changes in mental status. She was agitated, confused with visual hallucinations and combative behaviour. On day 4, she developed partial complex seizure with impairment of awareness and automatisms of lip smacking. During the first 2 weeks, she had become increasingly drowsy and confused, her level of conciousness was severely impaired, with no response to painful stimuli, and orofacial-limb dyskinesias appeared. These symptoms and central hypoventilation led to her being sedated on mechanical ventilation for 4 weeks. On day 31 after admission, a cardiac arrhythmia, characterized by alternating repeated episodes of tachycardia and bradycardia was observed.

The serum did not contain anti-nuclear, doublestrand DNA, SS-A or thyroid antibodies. Tumor markers were normal. Lumbar pucture on day 8 after the onset of symptoms showed lymphocytic pleocytosis $\left(22 / \mathrm{mm}^{3}\right)$ with normal protein and glucose concentrations. Cerebrospinal fluid cytology was negative for malignant cells. Anti-voltage-gated potassium channel complex antibodies were not detected.

Computed tomography of the chest, abdomen and pelvis was negative. Brain MRI with gadolinium-enhanced T1-weighted, T2-weighted, perfusion, diffusion and FLAIR images on days 2, 12, 31 and 63 were normal.

Intravenous immunoglobulin (IVIG) was given at a dose of $400 \mathrm{mg} / \mathrm{kg} /$ day for five days (days 31-36). By day 38 she had improved significantly, was awake and alert, and following commands without dyskinesias. On day 42, FDG-PET of the body was normal, but in the brain (Figure) showed increased metabolism in right hemisphere apparently in the territory of the middle cerebral artery and in the left cerebellum compatible with cerebellar diaschisis. NMDA receptor antibodies were positive.

NMDA receptor antibody encephalitis is a highly characteristic syndrome evolving in 5 stages, namely, the prodromal phase, psychotic phase, unresponsive phase, hyperkinetic phase, and gradual recovery phase, but only two clear stages have been supported with experimental data ${ }^{1}$. Temporal analysis of the onset of neurological features suggested progression through two main stages. The time of onset of the early features, characterized by neuropsychiatric symptoms and seizures preceded by a median of 10-20 days, the onset of movement disorders, reduction in consciousness and dysautonomia ${ }^{1}$. This disorder has often a relapsing course, therefore once patients overcome the hyperkinetic phase, gradual improvement happens in months and full recovery in 3 or more years.

Good clinical outcomes, as defined by reductions in modified Rankin scores, correlates with decreased NMDA receptor antibody levels and were associated with early ( $<40$ days) administration of immunotherapies in non-paraneoplastic patients.

NMDA receptor antibodies in serum and CSF are considered to be highly specific for this condition ${ }^{2}$. Recent studies show that this disorder can occur even in the absence of teratoma in up to $40 \%$ of patients, and even boys and adult men has been affected ${ }^{1}$.

In the $45 \%$ of patients the brain MRI is normal ${ }^{2}$. In our patient, brain FDG-PET managed to detect the abnormality, whereas MRI appeared normal on several occasions. Different patterns has been reported in NMDA receptor encephalitis, with hypermetabolism unilateral affectation of temporal lobe, brainstem and cerebellum ${ }^{3}$, bilateral primary motor, premotor, and supplementary motor areas, and temporal lobe and cerebellum crossed as in our patient. This crossed affectation is similar to
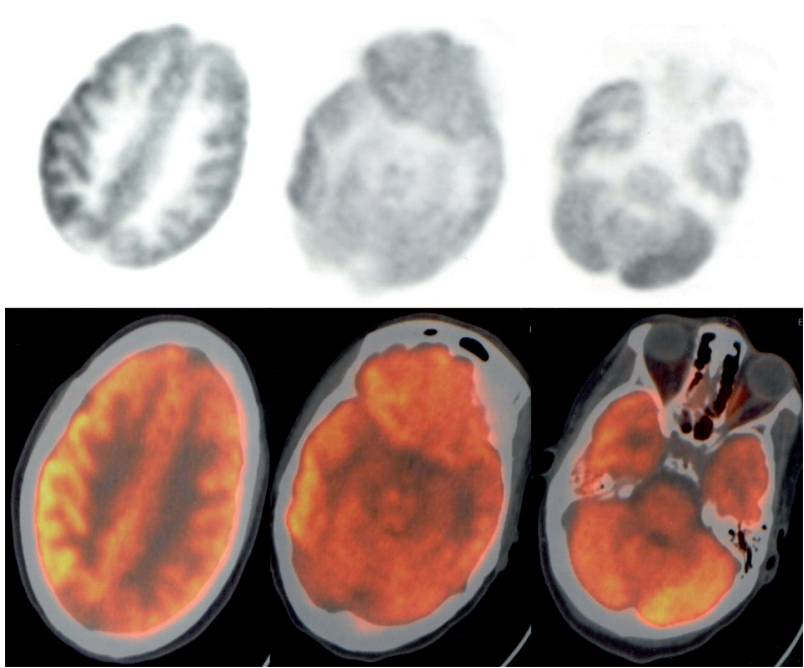

Figure. Brain FDG-PET showed increased metabolism in right hemisphere apparently in the territory of the middle cerebral artery and in the left cerebellum compatible with cerebellar diaschisis. The pattern is also seen in stroke. 
seen in stroke but with hypometabolism possibly representing a phenomenon of diaschisis. Serial FDG-PET examinations showed markedly increased activity in the basal ganglia as compared with that in the cortex when extrapyramidal features were prominent, which normalised after improvement of this movement disorder ${ }^{4}$. In the setting of paraneoplastic neurological syndrome, FDG-PET imaging can be of value from the following standpoints: [1] detection of the occult malignant focus and [2] objective assessment of the presence and extent of the functional abnormality in the brain and correlation of the imaging findings with the clinical features and disease activity ${ }^{5}$. The literature describing the central nervous system findings with PET in paraneoplastic neurological syndromes are primarily restricted to paraneoplastic limbic encephalitis. FDG-PET usually shows hypermetabolism in one or both temporal lobes, and the magnetic resonance imaging (MRI) findings correlate with the FDG-PET scan results in only a fraction of cases. FDG-PET images can provide additional clinical information which is of great value in further patient management.

In conclusions, the FDG-PET more often than the MRI revealed abnormalities in the brainstem, cerebral cortex or cerebellum, some of them clinically silent.

FDG-PET might be useful in the evaluation of patients suspected for NMDA receptor encephalitis, but studies to asses the utility for prognosis and treatment planning are necessary.

\section{REFERENCES}

1. Irani SR, Bera K, Waters P, et al. N-methyl-D-aspartate antibody encephalitis: temporal progression of clinical and paraclinical observations in a predominantly non-paraneoplastic disorder of both sexes. Brain 2010;133: 1655-1667.

2. Dalmau J, Tüzün E, Wu H, et al. Paraneoplastic Anti-N-methyl-D-aspartate receptor encephalitis associated with ovarian teratoma. Ann Neurol 2007;61:25-36

3. Vitaliani R, Mason W, Ances B, Zwerdling T, Jiang Z, Dalmau J. Paraneoplatic encephalitis, psychiatric symptoms, and hypoventilation in ovarian teratoma. Ann Neurol 2005;58:594-604.

4. Maeder-Ingvar M, Prior JO, Irani SR, Rey V, Vincent A, Rossetti AO. FDGPET hyperactivity in basal ganglia correlating with clinical course in antiNMDA-R antibodies encephalitis. J Neurol Neurosurg Psychiatry 2011;82: 235-236.

5. Basu S, Alavi A. Role of FDG-PET in the clinical management of paraneoplastic neurological syndrome: detection of the underlying malignancy and the brain PET-MRI correlates. Mol Imaging Biol 2008;10:131-137.

\section{EMISSÃO DE POSITRON FLUORODEOXYGLUCOSE NA ENCEFALITIDE DE} RECEPTOR ANTI-NMDA

Medical Doctor, Neurologist, Department of Neurology, Hospital Virgen de la Salud, Toledo, Spain.

Correspondence: Pedro Enrique Jiménez Caballero - Calle Dionisio Acedo no 9 / Portal 7, 4-1 - 10001 Cáceres - Spain. E-mail: pjimenez1010j@yahoo.es

Received 10 January 2011. Received in final form 7 February 2011. Accepted 14 February 2011.

\title{
Myasthenia gravis complicated with cryptococcal meningitis after thymectomy and long-term immunosuppressive therapy
}

\author{
Paulo J. Lorenzoni, Rosana H. Scola, Cláudia S.K. Kay, Sérgio M. Almeida, \\ Marisol D. Muro, Ismael P. Búrigo, Hipólito Carraro Jr, Lineu C. Werneck
}

Myasthenia gravis (MG) is an immune-mediated disease that compromises the postsynaptic membrane of the neuromuscular junction ${ }^{1,2}$. Common treatments for MG include immunosuppressive drugs and thymectomy ${ }^{1,2}$.

Cryptococcal meningitis (CM) is caused by the encapsulated yeast Cryptococcus neoformans, which is most notably known as an opportunistic infection in patients with human immunodeficiency virus (HIV) ${ }^{3}$. Development of CM is extremely rare in the HIV-negative population and is usually seen in the setting of diseases involving marked cell immunodeficiency ${ }^{3}$.

\section{CASE REPORT}

This report describes a 42-year-old woman who presented with intermittent diplopia, limited ocular movements, progressive eyelids ptosis and proximal limb weakness, which increased during periods of activity and decreased after periods of rest. The investigation yielded the following results: positive anti-acetylcholine receptor antibody test $(1.09 \mathrm{nmol} / \mathrm{l}$; normal $<0.20 \mathrm{nmol} / \mathrm{l})$; recordings of compound muscle action potential with an abnormal decrement of greater than 10\% during repetitive nerve stimulation; symptom improvement after 